\title{
Individualizing PSA Monitoring Among Older Prostate Cancer Survivors
}

\author{
Ying Shi, PhD ${ }^{1,2}$, Kathy Z. Fung, MS ${ }^{1,2}$, W. John Boscardin, PhD ${ }^{1,2,3}$, Sarah Ngo, MLIS ${ }^{1,2}$, \\ Stephen J. Freedland, MD ${ }^{4,5}$, Melisa L. Wong, $M D^{1,2,6}$, and Louise C. Walter, MD ${ }^{1,2}$
}

'Division of Geriatrics, San Francisco VA Health Care System 181G, San Francisco, CA, USA; ${ }^{2}$ Division of Geriatrics, University of California, San Francisco, San Francisco, CA, USA; ${ }^{3}$ Department of Epidemiology and Biostatistics, University of California, San Francisco, San Francisco, CA, USA; ${ }^{4}$ Center for Integrated Research on Cancer and Lifestyle, Samuel Oschin Comprehensive Cancer Institute, Cedars-Sinai Medical Center, Los Angeles, CA, USA; ${ }^{5}$ Division of Urology, Durham VA Medical Center, Durham, NC, USA; ${ }^{\circ}$ Division of Hematology/Oncology, University of California, San Francisco, San Francisco, CA, USA.

KEY WORDS: aging; survivorship; PSA monitoring; prostate cancer.

$\mathrm{J}$ Gen Intern Med 33(5):602-4

DOI: $10.1007 / \mathrm{s} 11606-018-4334-9$

(c) Society of General Internal Medicine (outside the USA) 2018

\section{INTRODUCTION}

Prostate cancer survivors are the largest group of male cancer survivors $(41 \%)$ and most are over age $65 .{ }^{1}$ Following curativeintent surgery or radiation, some data suggest interventions given shortly after early detection of biochemical recurrence (i.e., isolated prostate-specific antigen [PSA] elevation) may improve prostate cancer-specific survival. ${ }^{2}$ However, PSA monitoring may lead to complications from invasive diagnostics or treatment, and may be unnecessary in older men with a history of low-risk cancer or limited life expectancy due to the long lead time (5-8 years) between PSA biochemical recurrence and metastatic disease. ${ }^{2,3}$ Therefore, we examined national VA and Medicare data of older men treated for localized prostate cancer with radiation or radical prostatectomy to determine if PSA monitoring is more frequent among older men with substantial life expectancy and/or history of high-risk cancer, who are most likely to benefit, and less frequent among older men with significant comorbidity and/or history of low-risk cancer who are most likely to experience harms without benefit.

\section{METHODS}

We used the VA Central Cancer Registry to conduct a longitudinal cohort study of 13,397 men age 65 or older who were newly diagnosed with clinically localized prostate cancer between $1 / 1 / 03$ and $12 / 31 / 08$, received initial curative-intent radical prostatectomy or radiation, and survived without biochemical recurrence for at least 1 year after treatment. All men were followed for 4 years after their 1-year treatment anniversary date using national VA and Medicare data. We excluded men who did not

Published online February 7, 2018 have VA or Medicare as their primary insurance and those enrolled in Medicare HMO during the study period because they lacked claims to determine comorbidities or PSA monitoring.

Our main outcome was the number of PSA tests performed per person-year across all four survivorship years and for each survivorship year separately. For example, survivorship year 1 was defined as days $365-729$ posttreatment to avoid including PSA tests performed during or immediately after treatment as most men would have completed treatment within a year.

We used Poisson regression models to estimate the number of PSA tests performed per person-year for baseline patient and tumor characteristics, with censoring at time of biochemical recurrence or death. Death was determined by the VA Vital Status file.

\section{RESULTS}

Baseline characteristics and associated PSA monitoring frequencies are presented in Table 1. No tumor or patient characteristic was associated with greater than 2.1 PSA tests per person-year or less than 1.8 PSA tests per personyear. Similarly, within each cancer recurrence risk group, men with limited life expectancy received slightly more PSA tests per person-year compared to men with favorable life expectancy (overall $P<0.001$ ) (Fig. 1). Also, men with limited life expectancy treated for low-risk cancer, who are least likely to experience prostate cancer recurrence in their life time, received 1.9 PSA tests per person-year. This is only slightly lower than men with favorable life expectancy treated for high-risk cancer, who received 2.0 PSA tests per person-year $(P<0.001)$.

Also, predictors of PSA monitoring were generally consistent within each survivorship year. However, the number of PSA tests decreased from 2.3 PSA tests per person-year in survivorship year 1, to 2.0 in year 2, 1.8 in year 3, and 1.6 in year 4 ( $P<0.001$ for trend). 
Table 1 Baseline Characteristics and Frequency of PSA Monitoring for Recurrence During Survivorship Years 1-4 (N=13,397 Men Aged 65 Years or Older Treated for Localized Prostate Cancer with Curative-Intent*)

\begin{tabular}{|c|c|c|}
\hline Characteristics & $n(\%)$ & $\begin{array}{l}\text { Frequency of PSA monitoring: } \\
\text { Number of PSA tests per person-year } \\
\text { ( } 95 \% \text { confidence interval) }\end{array}$ \\
\hline \multicolumn{3}{|l|}{ Age at diagnosis } \\
\hline $65-69$ years & $5952(44.4)$ & $1.95(1.93,1.97)$ \\
\hline $70-74$ years & $4795(35.8)$ & $1.94(1.92,1.96)$ \\
\hline $75+$ years & $2650(19.8)$ & $1.90(1.87,1.93)$ \\
\hline \multicolumn{3}{|l|}{$\operatorname{Race}^{\dagger}$} \\
\hline White & $10,300(77.3)$ & $1.93(1.91,1.94)$ \\
\hline Black & $2629(19.7)$ & $2.00(1.97,2.03)$ \\
\hline Other & $394(3.0)$ & $1.95(1.88,2.02)$ \\
\hline \multicolumn{3}{|c|}{ Charlson Comorbidity Score $(\mathrm{CCI})^{\ddagger}$} \\
\hline 0 & $6385(47.7)$ & $1.90(1.88,1.92)$ \\
\hline $1-3$ & $5533(41.3)$ & $1.97(1.95,1.99)$ \\
\hline $4+$ & $1479(11.0)$ & $2.02(1.97,2.06)$ \\
\hline \multicolumn{3}{|c|}{ Lived in ZCTA in which $\geq 25 \%$ of adults had a college education } \\
\hline No & $9310(72.0)$ & $1.93(1.92,1.95)$ \\
\hline Yes & $3617(28.0)$ & $1.94(1.92,1.97)$ \\
\hline \multicolumn{3}{|c|}{ Primary insurance at diagnosis } \\
\hline VA & $9940(74.2)$ & $1.92(1.90,1.93)$ \\
\hline Medicare & $3457(25.8)$ & $2.00(1.97,2.02)$ \\
\hline \multicolumn{3}{|c|}{ Year of prostate cancer diagnosis } \\
\hline $2003-2005$ & $6900(51.5)$ & $1.94(1.92,1.96)$ \\
\hline $2006-2008$ & $6497(48.5)$ & $1.94(1.92,1.95)$ \\
\hline \multicolumn{3}{|l|}{ Type of treatment received } \\
\hline Prostatectomy & $3578(26.7)$ & $1.81(1.79,1.83)$ \\
\hline Radiation & 9819 (73.3) & $1.98(1.97,2.00)$ \\
\hline \multicolumn{3}{|c|}{ Prostate cancer recurrence risk based on tumor characteristics ${ }^{\dagger \dagger 11}$} \\
\hline Low-risk cancer & $4195(32.1)$ & $1.84(1.82,1.86)$ \\
\hline Intermediate-risk cancer & $4510(34.5)$ & $1.94(1.92,1.96)$ \\
\hline High-risk cancer & $4358(33.4)$ & $2.06(2.04,2.09)$ \\
\hline \multicolumn{3}{|l|}{ Life expectancy ${ }^{\text {II }}$} \\
\hline Limited & $1516(11.3)$ & $2.00(1.96,2.04)$ \\
\hline Intermediate & $6629(49.5)$ & $1.95(1.93,1.97)$ \\
\hline Favorable & $5252(39.2)$ & $1.91(1.89,1.93)$ \\
\hline
\end{tabular}

*All men survived without biochemical recurrence for at least 1 year after treatment. Biochemical recurrence following radical prostatectomy was defined as the date of an initial PSA level $\geq 0.2 \mathrm{ng} / \mathrm{ml}$ that was confirmed by a second PSA level $>0.2 \mathrm{ng} / \mathrm{ml}$. Biochemical recurrence after radiation was determined using the Phoenix definition (i.e., date of first PSA level exceeding the PSA nadir $+2 \mathrm{ng} / \mathrm{ml}$ )

+ Missing values: race, 0.6\%; Zip Code Tabulation Area (ZCTA) college education, 3.5\%; prostate cancer recurrence risk based on tumor characteristics, $2.5 \%$

$¥$ The Charlson Comorbidity Index (CCI) is a measure of 19 chronic diseases weighted according to their association with mortality. CCI was calculated using VA and Medicare inpatient and outpatient claims during the 12 months prior to the beginning of survivorship year 1. We excluded the diagnosis of prostate cancer from the CCI for all patients to focus on non-prostate cancer comorbidity predictive of life expectancy separate from tumor characteristics

${ }^{\xi}$ We used linkages to the 2000 US Census to determine for each veteran's ZIP Code Tabulation Area (ZCTA) the percentage of adults with a college education

IIProstate cancer recurrence risk based on tumor characteristics was calculated based on Gleason grade, clinical stage, and PSA level at diagnosis (see Fig. 1 footnote for definitions of the three mutually exclusive cancer risk groups) ${ }^{4}$

"Life expectancy was calculated based on age and CCI (see Fig. 1 footnote for definitions of the three mutually exclusive life expectancy groups). Men with limited life expectancy have $>50 \%$ 5-year mortality

${ }^{\#}$ PSA monitoring within the VA healthcare system was defined by a PSA in the LAR dataset during survivorship years 1-4. LAR extracts all PSA results from VA facilities. PSA monitoring within the Medicare system was identified by Current Procedure Terminology codes G0103 and 84153

\section{DISCUSSION}

We found most men received approximately two PSA tests per year over survivorship years $1-4$, which is consistent with national guidelines and two earlier studies. ${ }^{5,6}$ However, prior studies did not include men treated with radiation or determine PSA monitoring according to life expectancy or tumor characteristics. We found a narrow range of PSA monitoring frequencies across patient and tumor characteristics, indicating little individualization of PSA monitoring. The strongest predictor of decreased PSA monitoring was time since treatment.
This is the largest national study to determine the use of PSA monitoring in older prostate cancer survivors following curative-intent surgery or radiation. Limitations include lack of PSA results in the $10 \%$ of tests performed in Medicare. Also, generalizability of our findings to non-veterans is uncertain. However, the VA is the largest US healthcare system. Our findings suggest a need for guidelines to encourage PSA monitoring as an informed decision that considers life expectancy, risk of recurrence and values/preferences of cancer survivors rather than a one-size-fits-all approach. 


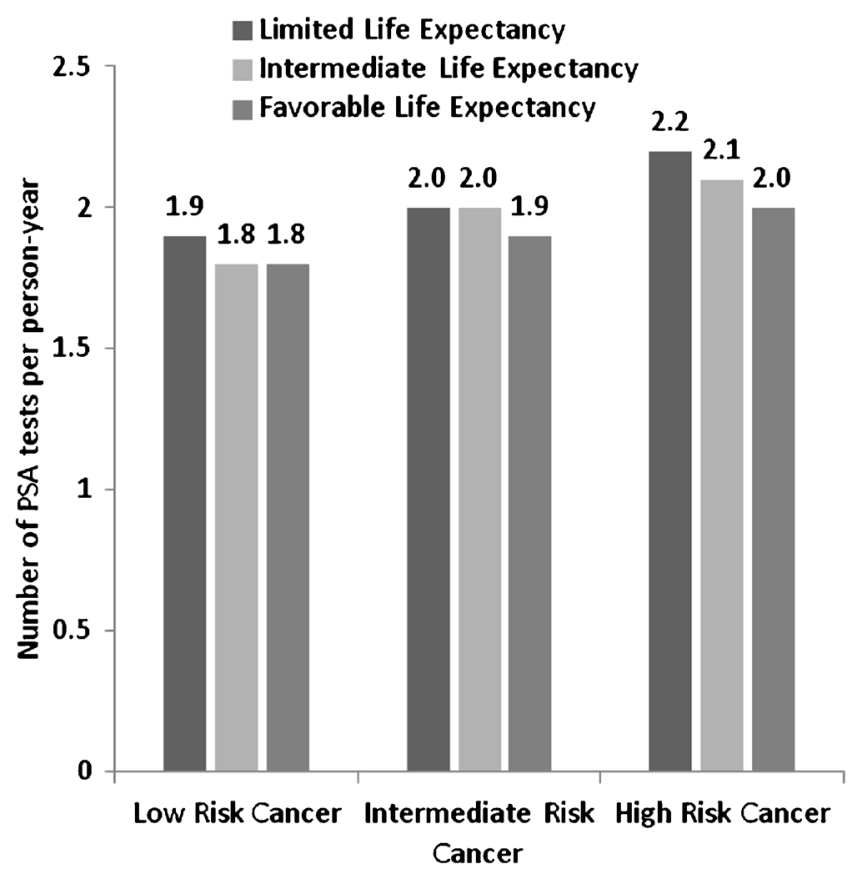

Figure 1 Frequency of PSA monitoring during survivorship years $1-4$, according to tumor characteristics predictive of recurrence risk ${ }^{\dagger}$ and patient characteristics predictive of life expectancy ${ }^{*}{ }^{\dagger}$ Tumor characteristics: Low-risk cancer $=$ stage $\leq$ T2a, PSA level $\leq 10 \mathrm{ng} /$ $\mathrm{ml}$, and Gleason score $\leq 6$; intermediate-risk cancer $=$ stage $\mathbf{T} 2 \mathrm{~b}$, or PSA level 10.1-20 $\mathrm{ng} / \mathrm{ml}$ or Gleason score $=7$; and high-risk cancer $=$ stage T2c-T3b or PSA level $>20 \mathrm{ng} / \mathrm{ml}$ or Gleason score $\geq 8 .{ }^{4}$

*Patient characteristics: limited life expectancy $=$ men aged 85 years and older with Charlson Comorbidity Index $(\mathrm{CCI}) \geq 1$ or those aged 65 years and older with $\mathrm{CCI} \geq 4$; favorable life expectancy $=$ men aged 65-74 years with $\mathrm{CCI}=0$; and intermediate life expectancy $=$ everyone else.

Corresponding Author: Louise C. Walter, MD; Division of GeriatricsSan Francisco VA Health Care System 181G, 4150 Clement Street, San Francisco, CA 94121, USA (e-mail: Louise.Walter@ucsf. edu).
Funding Information This work was supported by the National Institute on Aging at the National Institutes of Health (grant number K24AG041180) to [LW].

The funding sources had no role in the design, conduct, or analysis of this study or in the decision to submit the manuscript for publication.

\section{Compliance with Ethical Standards:}

The Committee on Human Research at the University of California, San Francisco, and the Committee for Research and Development at the San Francisco VA Medical Center approved this study.

The corresponding author, Louise Walter, had full access to all of the data in the study and takes responsibility for the integrity of the data and the accuracy of the data analysis. The datasets generated during and/or analyzed during the current study are not publicly available because Veterans Affairs data are not publicly available. However, all datasets used in this study are available to VA investigators through VIReC, http://vaww.virec.research.va.gov/Index-VACMS.htm

Conflict of Interest: The authors declare that they do not have a conflict of interest.

\section{REFERENCES}

1. Siegel RL, Miller KD, Jemal A. Cancer statistics, 2016. CA Cancer J Clin. 2016;66(1):7-30.

2. Trock BJ, Han M, Freedland SJ, et al. Prostate cancer-specific survival following salvage radiotherapy vs observation in men with biochemical recurrence after radical prostatectomy. JAMA 2008;299(23):2760-2769.

3. Pound CR, Partin AW, Eisenberger MA, Chan DW, Pearson JD, Walsh PC. Natural history of progression after PSA elevation following radical prostatectomy. JAMA 1999;281(17):1591-1597.

4. Shao YH, Albertsen PC, Roberts CB, et al. Risk profiles and treatment patterns among men diagnosed as having prostate cancer and a prostatespecific antigen level below $4.0 \mathrm{ng} / \mathrm{ml}$. Arch Intern Med. 2010;170(14):1256-1261.

5. Elston Lafata J, Simpkins J, Schultz L, et al. Routine surveillance care after cancer treatment with curative intent. Med Care. 2005;43(6):592599.

6. Trantham LC, Nielsen ME, Mobley LR, Wheeler SB, Carpenter WR, Biddle AK. Use of prostate-specific antigen testing as a disease surveillance tool following radical prostatectomy. Cancer 2013;119(19):3523-3530. 